\title{
Recent Research Progress on Study of Paragonimus westermani in China
}

\author{
Dingli Xu, Jing Li, Qi Liao, Tuergong Jiang, Jiang Cai, Yuelong Lv, Jiakuan Lv, Baile Shen, \\ Zexian Xu, Yanting Zhang, Jianfa Liu* \\ Ningbo University School of Medicine, Ningbo, China \\ Email: "liujianfa@nbu.edu.cn
}

Received 20 April 2015; accepted 11 May 2015; published 18 May 2015

Copyright $@ 2015$ by authors and OALib.

This work is licensed under the Creative Commons Attribution International License (CC BY).

http://creativecommons.org/licenses/by/4.0/

(c) †) Open Access

\begin{abstract}
Recently, along with the development of biochemical and molecular immunological techniques, the diagnosis methods have been improved in which preparing natural parasite antigens, and genetically engineered recombinant antigens have been applied by using agglutination test, complement fixation methods to detect the human's serum antibodies for parasite identification of paragonimus infections. To improve the detection rate, some scholars have used phage peptide library technology to further improve the diagnostic effect on Paragonimus westermani in humans. In this paper, we summarized recent studies on diagnosis, pathogenesis and treatment of Paragonimus flukes in China.
\end{abstract}

\section{Keywords}

Paragonimus westermani, Antigen, Phage Peptide Library Technology, China

Subject Areas: Infectious Diseases, Respiratory Medicine

\section{The Outline of Paragonimus westermani}

\subsection{Morphological Characters}

The ovum of Paragonimus westermani is oval in shape and golden yellow in color and its operculum is big, conspicuous and inclined usually. Its ootheca is near papery and the other side is incrassate. There is an egg cell in the center of ovum surrounded by several yolk cells.

The imago looks like a half peanut, the reverse side of which is upheaval and the abdomen sucker is planar, while the size of oral sucker is similar to ventral sucker. Every adult has two intestinal tubes, arranging along the two sides of polypide. The ovary and uterus are tied behind the ventral sucker, meanwhile the testicular is di-

${ }^{*}$ Corresponding author.

Financially Supported by Natural Scientific Foundation of Ningbo City (2010A610036) and Subject Programme Foundation of Ningbo University (XKL14D2105)

How to cite this paper: Xu, D.L., Li, J., Liao, Q., Jiang, T., Cai, J., Lv, Y.L., Lv, J.K., Shen, B.L., Xu, Z.X., Zhang, Y.T. and Liu, J.F. (2015) Recent Research Progress on study of Paragonimus westermani in China. Open Access Library Journal, 2: e1511. 
vided into two and tied in polytide after a third position [1].

\subsection{Life Cycle Characters}

The life cycle of Paragonimus westermani includes ovum, miracidium sporocyst, redia, cercaria, metacercariae and adults. When the ovum passed into water, it would grow and mature under appropriate environment for almost three weeks, then the miracidium would be hatched from ovum. The worm would invade into the first intermediate host, then, the miracidium would live through the phase of sporocyst and redia, and finally develop into cercaria, which then escape from the snails and intrude into the body of second intermediate host, such as freshwater crab or crayfish in which it grow into a mature metacercariae. People would often be infected if they ate the raw freshwater crab or crayfish containing living metacercariae which would take off the capsule with the help of decomposing of digestive enzyme in small intestine, then enter into pulmonary across the diazoma. It would mature and form cyst in the pulmonary finally called paragonimiasis. The immunopathological effect caused by the migration and the parasitism of paragonimiasis was the primary mechanism of pathopoiesis [2].

\subsection{Symptoms and Signs}

Patients at acute stage would appear some symptoms such as chilly, remittent fever, bellyache, diarrhoea, feeblity, night sweat and anorexia during a week of infection. Then it would appear pectoralgia, cough, asthma in two or three weeks. On the other hand, the chronic typical symptoms were cough and hemoptysis [3]. The sputum was jam-like where we can find Charcot Leyden crystals and plenty of ovums. In addition, the chronic phage could be splited into various types according to its basic symptom and pathogenesis. For example, the basic pathology of the pulmonary type was lung abscess and hydatoncus in which we could find too many ovums. A few number of patients would appear expiratory dyspnea, asthma and pleural effusion. The clinical manifestations of abdominal type were abdominal pain, diarrhoea, vomit and hematochezia, the severe abdominal pain such as acute abdomen was rare. The intestinal type mainly demonstrates abdominal pain, diarrhoea and mucous bloody stool. The patients of cerebral type or myeloid form would appear some changes in cellular damages. The number of cells and proteins would increase slightly and the eosinophile granulocyte appeared. In addition, these patients' intracranial pressure would rise too quickly. Above all, the chronic patients have common characteristics in which the old and new lesions alternate each other due to the wandering of larvae and imago.

\section{The Diagnosis and Identification of Paragonmus westerman}

Previous morphological methods on parasitic detection with the electron microscope are used for identifications of eggs and adults of Paragonimus westermani, even though the specificity is high, and the sensitivity is relatively low. Cultivation of the adult worms is also effective upon time, temperature, humidity and other restrictions. In addition, the shape characteristics of worms are similar to accurate identification [3]. On the immunological aspects, the paragonimus antigens used for clinical diagnosisis are basically same. It was very difficult to implement the large-scale survey of diseases only by pathogen detections. At the same time, the antigen complex will also affect the diagnosis results [4]. Some researchers also used the adult worm's cDNA library of Paragonimus westermani to screen out the positive antigens because of its high specificity and high sensitivity that provides the means for the diagnosis and treatment of infection with detection technology.

\subsection{Antigen Detection}

Antigens of lung fluke tested include circulating antigen (CAg) and circulating immune complexes (CIC). It is very helpful to clinically diagnose patients in endemic areas using CAg-dot-ELISA and CAb-ELISA methods. Some parasitologists had used CAg-dot-ELISA to detect 70 cases of patients with Paragonimus trematodes that has een confirmed that 29 cases were positive with sensitivity of $41.5 \%$ comparing with Clonorchis trematodes. Using CAb-ELISA to detect 70 cases of Paragonimus trematodes, 67 patients appeared positive with the sensitivity as $95.7 \%$ comparing with clonorchiasis diseases. CAg-dot-ELISA method was also used for the early diagnosis of Paragonimus infections because of CAg appearing earlier, at that time, a low level of antibodies had not yet been detected [5]. For CAg detection, early diagnosis can also be used as indicators of assessment activity and therapeutic effects of infections. Dogs infected with Paragonimus westermani metacercariae isolated from crabs would be quantitatively diagnosed with multi-resistant dot-EusA assay [6]. 


\subsection{Antibody Detection}

Using intradermal test with 1: $0.1 \mathrm{ml}$ antigen to detect adults' paragonimiasis, the positive rate is up to $95 \%$. The method is simple, but the specificity is not high, and there exists a higher cross-reactivity among schistosomiasis and chlonochiasis which would be used for epidemiological screenings [7]. After using cercariae membrane reaction to test metacercariae that were incubated in serum, the sensitivity is $98.5 \%$ and $100 \%$ with the value of early diagnosis, but there is a positive cross-react compared with schistosomiasis and fasciolopsiasis, etc. [7].

Currently, rapid detection methods for paragonimiasis include using P-IgG4-DIGFA for indication, the sensitivity and specificity were $95.2 \%$ and $100 \%$ respectively. Gold filtration assay for rapid detection of specific IgG4 of lung flukes is not only simple, fast, but also high sensitivity and specificity which is suitable for clinical laboratory and field investigation applications [8].

\subsection{Cross-Immunity Reaction}

Paragonimiasis and paragonimus infections could be identified by SDS-PAGE, spectrometry detection and immuno-gold labeling that showed the main components of the parasite surface membrane and secretions [9] [10].

\subsection{Advances in Molecular Biology}

Genetic screening and expression of recombinant antigens of Paragonimus westermani were screened by ELISA and cell cultures. Performed with Paragonimus westerman, the cDNA library was screened five times and the positive clones were amplified by PCR using in vivo phage of recombinant plasmids of DNA sequence determination. BLAST method (accounting sequence database search program) was used to detect DNA sequence homology that may act as a Paragonimus westermani's gene fragment of positive clones [11]. Various developmental stages of Paragonimus westerman secrete at least six different types of cysteine proteases, in nutritional intake, invasion, and immune evasion and has played an extremely important role in pathogenicity of the host in order to design targeted at the protein corresponding anti-infective drugs. Using cDNA library of Paragonimus westermani to screen recombinant bacterium gene of PwAg, the homology is as high as 99\% [12]. Phage screening method on Paragonimus antigen epitope of simulation can also be helpful for the diagnosis. The new diagnostic reagents provide a new focus from cDNA screening [13]. For patients with lung fluke, their antibody reactions with the existing detection methods could be extracted serum antigen that does not require cultured parasites [14].

\subsection{The Genetic Testing of Paragonimus westermani}

Ribosomal DNA gene and a second section (ITS2) sequences of mitochondrial cytochrome C oxidase subunit (COI) partly on Paragonimus westermani had been studied and colonizated. COI gene belongs to the mitochondrial genome, its evolutionary rate was faster than the nuclear genome, and with maternal inheritance, it was basically not affected by genetic recombination, genetic differences within species. ITS2 gene evolution rate is relatively slow and conservative, reflecting the level of variation in parasites suitable for Paragonimus genetic differences between species, especially for close genetic relationship and is the ideal targets for detection and identification of parasites. In 1970s, Japanese researchers found that Paragonimus had two types of genotyping such as triploid $(2 n=223 n=33)$ which was very similar to the metacercariae, worms and eggs. Diploid type worms have sperms, the reproductive mating lines, known as Paragonmus westermani. Triploid type worms have no sperms, but pathogenic impacts are significantly stronger than diploid of Paragonimus [15]. According to specific gene sequences (ITS2) of Paragonimus westermani, parasitologists had designed specific PCR primers and probes suitable for the detection of paragonimiasis in order to distinguish Paragonimus westermani. from $\mathrm{Pa}$ ragonimus westermani, Paragonimus Okazaki, Mexico Paragonimus, Fasciollopsis, liver flukes and Schistosomas using the second rRNA and the internal transcribed spacer District ITS2 gene sequences. In specific PCR testing method, only Paragonimus westermani significant DNA samples have amplified bands, and in the specific real-time PCR testing method, it is only Paragonimus westermani that appear fluorescent amplification curves.

\section{Prospect}

Excluding diagnosis on lung flukes and identification of patients experienced more than parasites with electron 
microscopy and optical microscopy, identifications of antigen-antibody agglutination test and other immunological methods were used and developed. Due to limitations of parasites' antigens, a large-scale preparation of recombinant antigens and improving its purity would enter a new field conducted in-depth research. Positive clones have been obtained with immunological method for Paragonimus cDNA library by PCR amplification which addressed the questions of quantity and improved the specificity and accuracy of detections. At the genetic levels, specific primers and probes designed by PCR or real-time PCR methods for identification of similar paragonimus species are made as possible.

\section{References}

[1] Chen, S.H., Zhou, X.-N. and Zhang, Y.-N. (2007) Dynamics of Specific Antibody and Circulating Antigen in Sera from the Dogs Infected with Paragonimus westerman. Chinese Journal of Veterinary Parasitology, 15, 11-14.

[2] Chen, Q.J. and Yin, J.-G. (2007) Chin Research and Perspectives in Parasitology. Chinese Journal of Parasitology \& Parasitic Diseases, 25, 344-348.

[3] Zhang, D., Wang, Z.Q. and Cui, J. (2003) The Specific Diagnostic Antigens of Paragonimus westermani Adult Worms. The Jounal of Zhenzhou University, 38, 538-540.

[4] Li, T.-Q., Jiang, C.-F. and Shi, H.-B. (2005) Observation on the Immuno-Reactivity of the Mimic Antigen Epitope of Paragonimus westermani. Chinese Journal of Zoonoses, 21, 507-509.

[5] Chen, S.-H., Li, H. and Zhou, X.-N. (2007) Establishment and Application of Circulating Antigen Detection in Paragonimus westermani Infection. Chinese Journal of Parasitology \& Parasitic Diseases, 25, 513-514.

[6] Lin, Z.-G., Li, Y.-S. and Zhang, Z.-P. (2003) Investigation on Paragonimiasis in Yanping County of Fujian Province. Journal of Tropical Diseases and Parasitology, 1, 135-137.

[7] Ji, H.-J. and Wang, H., ( 2012) The Diagnosis and Treatment of Pleural Effusion of Paragonimiasis. Journal of Clinical Pulmonary Medicine, 17, 1872-1874.

[8] Tong. D.S., Liu, Y.X. and Tang, F. (2013) Detection of Four Parasitic Infections Using ELISA. Chinese Journal of Schistosomiasis Control, 25, 327-328.

[9] Ma, A., Shi, X.-H., Wang, Y. and Gan, X.-X. (2005) Development and Application of Rapid Detection in Specific IgG4 of Paragonimiasis. Chinese Journal of Zoonoses, 26, 353-355.

[10] Niu, J.-X., Zhang, X.-L. and He, X. (2010) Adult Antigens of Pagumogonimus skrjabini Identified by the Serum with Paragonimiasis and Analysis on Their Characterizations. International Journal of Medical Parasitic Disease, 37, 136-140.

[11] Lin, J.-J., Zhang, Z.-H. and Zhang, Y.J. (2002) Immunoscreening of Paragonimus westermani Adult cDNA Library Using Monoclone Antibodies. Chinese Journal of Zoonoses, 18, 48-53.

[12] Liu, J.-N., Ling, J.-J., Hou, M., Zhang, Z.-H. and Zhang, Y.-J. (2003) The Experience and Identification of Paragonimlasis westermani Adult Recombinant Antigen. Chinese Journal of Parasitology and Parasitic Disease, 16, 96-98.

[13] Lei, J.-H., Jiang, C.-F. and Li, T.-Q. (2004) Comparing the Antigenicty of Two Kinds of Antigen-Mimic Epitopes of Paragonimus westermani Screened from the 12-Mer Phage Peptide Library Used Different Methods. Journal of Tropical Diseases and Parasitology, 2, 3-6.

[14] Liu, J.-F., Zhang, J.-N. and Jiang, W.-W. (2013) Advance in Comparative Study on Diploid and Tripoid of Paragonimus wastermani. Chinese Journal of Zoonoses, 29, 191-194.

[15] Zhao, X., Zheng, Q.-Y., Cao, J.-J. and Zhao, Q.-C. (2008) Detection of Paragonimus westemrani by PCR and RealTime PCR. Biotechnology Bulletin, S1, 359-361. 